\title{
Comparison of analgesic efficacy after major surgery in pancreato-biliary cancers: Intravenous patient-controlled analgesia versus patient-controlled epidural analgesia
}

\author{
Jangho PARK ${ }^{1}$, Sang-Jae PARK ${ }^{* 1}$, Sung-Sik HAN ${ }^{1}$, Hyeong Min PARK'1, Eun Young PARK², Dae-Hyun KIM³, Soon-Ae LEE ${ }^{3}$, Sun-Whe KIM'1 \\ ${ }^{1}$ Center for Liver \& Pancreato-Biliary Cancer, National Cancer Center, Goyang, Korea \\ ${ }^{2}$ Biostatistics Collaboration Team, Research Core, Research institute, National Cancer Center, Goyang, Korea \\ ${ }^{3}$ Department of Anesthesiology and Pain Medicine, National Cancer Center, Goyang, Korea
}

Introduction: Postoperative pain relief is important to recover to normal life after abdominal surgery. Patient-controlled epidural analgesia (PCEA) has been reported to be more effective in the pain control than intravenous patient-controlled analgesia (IV-PCA). The purpose of this study was to compare outcomes including the effect of pain control between IV-PCA and PCEA in patients undergoing surgical resection of pancreato-biliary malignancies.

Methods: Between January 2018 and February 2020, 110 patients scheduled for major resection of pancreato-biliary cancers were enrolled to the randomized controlled trial and each of 55 patients was randomly assigned to PCEA group and IV-PCA group. Pain scales during resting on the day 2 postoperatively and during rest 3 times a day from the day 1 to 7 postoperatively were collected through the questionnaire. We invested serum level of troponin I on the day 1 postoperatively, postoperative complications, and PCA-related complications.

Results: Fifty-two patients in IV-PCA group and 50 patients in PCEA group were finally analyzed for the outcomes. There were no significant differences in the pain scales between the two groups during ambulation on the day 2 postoperatively and during rest from the day 1 to 7 postoperatively. No differences presented in serum level of troponin I on the day 1 postoperatively, postoperative complications, and total PCA-related complications.

Conclusions: PCEA has no superiority to IV-PCA on the pain relief after operation for pancreato-biliary malignancies. There were no significant differences in the postoperative outcomes including postoperative morbidity and PCA-related complications between the two groups. 